\title{
Principles of Phase Reconstruction with Aberration Correction Using Three-Dimensional Fourier Filtering Method
}

\author{
T. Kawasaki and Y. Takai
}

Department of Material and Life Science, Graduate School of Engineering, Osaka University, 2-1 Yamadaoka, Suita, Osaka 565-0871, Japan

Phase reconstruction is important for quantitative analysis of atomic structures in high-resolution transmission electron microscopy (HRTEM). Various methods have been developed thus far for phase reconstruction, such as electron holography and image processing using plural images taken at different focus conditions[1-2]. As one of the promising approaches, we have been proposed three-dimensional Fourier filtering method (3D-FFM)[3-4]. In present paper, reconstruction procedures of a phase image by the proposed method are introduced, and the effectiveness of this technique is confirmed in an experiment using an amorphous thin film.

Figure 1 is a schematic diagram of reconstruction process of a phase image with aberration correction. First, a 3D Fourier transform is performed to a recorded digital data set of through-focus images (Fig. 1(a)). In the 3D Fourier spectrum obtained by the Fourier transform, the structural information of the sample is localized on so-called twin Ewald spheres (Fig. 1(b)), which is formed by the linear interference components between the transmitted wave and the diffracted waves. The effect of aberrations of image-forming lenses then appears as the phase shift of each component on the twin spheres. To extract the required component, an appropriate filter function should be multiplied for the 3D Fourier spectrum (Fig. 1(c)). This filter function consists of a narrow band pass filter to extract the linear imaging component with a phase correction factor to eliminate the effect of aberrations. Finally, a phase image is reconstructed as one of the processed through-focus images by performing a 3D inverse Fourier transform to the corrected 3D Fourier component (Fig. 1(d),(e)).

To confirm the effect of phase reconstruction with simultaneous correction of spherical aberration and astigmatism, the 3D-FFM is performed to 256 through-focus images taken in every $3.75 \mathrm{~nm}$ focus step of an amorphous thin film ( $\sim 5 \mathrm{~nm}$ thickness, carbon and tungsten double-layered structure film), which contains structural information in a wide and continuous spatial frequency range. Figs. 2 and 3 show original and phase-reconstructed images with corresponding diffractograms, respectively, which are taken at three focus conditions ((a)? $\mathrm{f}=-77 \mathrm{~nm}$, (b)0nm, and (c) $+77 \mathrm{~nm}$ ). Astigmatism and spherical aberration is obviously contained in the original images, which can be recognized by the elliptical ring pattern in Fig. 2(a) and the difference between the 
diffractograms taken at the same absolute value of focus (Figs. 2(a) and (c)). In contrast, Figs. 3(a) and (c) represent the same circular ring pattern and the same image contrast with each other as shown by arrows. Furthermore, Fig. 3(b) shows a uniform round shape pattern with no oscillation, indicating that the phase information was retrieved in a wide range of spatial frequency by the successful correction of the residual aberrations.

\section{References}

[1] T. Ikuta, J. Electron Microsc. 38 (1989) 415

[2] D. Van Dyck, M. Op de Beeck and W. Coene, Optik 93 (1993) 103

[3] T. Kawasaki, Y. Takai, T. Ikuta, and R. Shimizu, Ultramicroscopy 90 (2001) 47-59

[4] T. Kawasaki, Y. Takai, and R. Shimizu, Applied Physics Letters 79(2001) 3509-3511

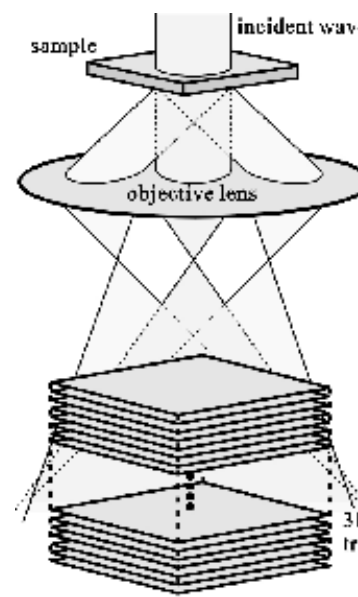

(a) Original through-focus image

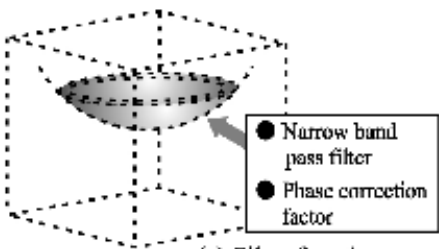

(c) Filter function

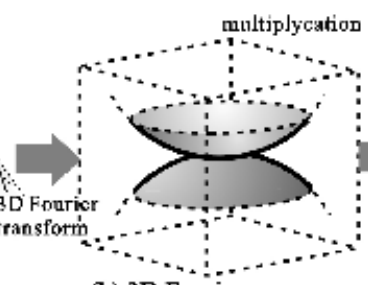

(b) 3D Fourier spectrum

Fig.1 Schematic diagram of reconstruction processes of a wave field
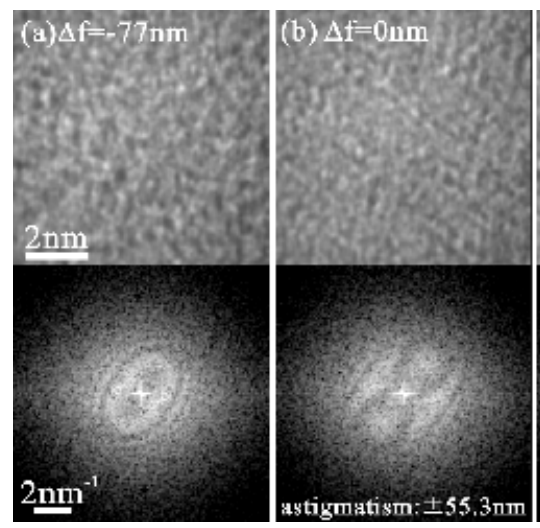

Fig.2

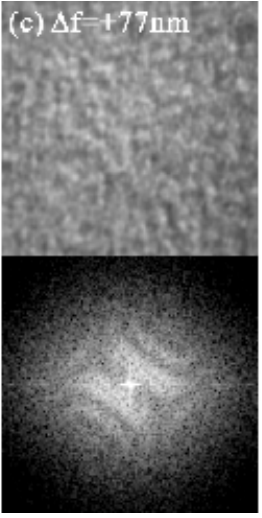
of an amorphous thin film, taken at three different defocus condition.
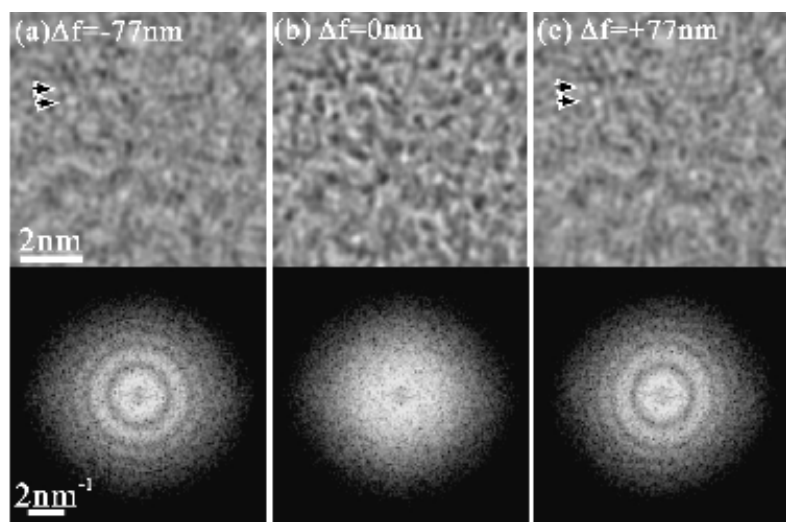

Fig.3 Processed images and corresponding diffractograms reconstructed from 256 through-focus images 